\title{
DIELECTRIC \& PHYSIOCHEMICAL CHARACTERIZATION OF NITROMETHANE WITH DMSO AT 293.15 K
}

\author{
ARUNA P. MAHAROLKAR I*, A. G. MURUGKAR ${ }^{2}, P$ W. KHIRADE ${ }^{2}$ \\ ${ }^{1}$ Marathwada Institute of Technology, Aurangabad, (M.S.) -431004, India \\ ${ }^{2}$ Department of Physics, Dr. B. A. M. University, Aurangabad, (M.S.) -431004, India
}

\begin{abstract}
The present paper reports study of dielectric \& physicochemical properties like dielectric constant, viscosity, density and refractive index for the binary mixtures of Nitromethane (NM) and Dimethylsulphoxide (DMSO) over the entire concentration range were measured at 293.15K. The experimental data further used to determine the Bruggeman factor, and excess properties viz. excess density, excess viscosity, and excess refractive index. The values of excess properties further fitted with Redlich-Kister equation. Excess properties were used to indicate the presence of intermolecular interactions and strength of intermolecular interactions between the molecules in the binary mixtures
\end{abstract}

Key words: excess static dielectric constant, R-K fit equation

\section{INTRODUCTION}

Liquid mixtures are pre-requisite in almost all industries and all chemical as well as biological sciences. Imperative attention is needed to understand these systems and to predict their behaviour from the molecular point of view[1-2]. Dielectric constant, Density, viscosity and refractive index of liquid mixtures mostly required in most engineering calculations and chemical, pharmaceutical industries where fluid flow of mixture is an important factor. Knowledge of the dependence of densities and viscosities of liquid mixtures on composition is of great interest from a theoretical stand point since it may lead to better understanding of the fundamental behaviour of liquid systems. Also it plays vital role in the design processes involving chemical separations, equipment design, heat transfer, fluid flow and molecular dynamics. Viscosity data are useful for testing theories $[3,5]$ we report here the Dielectric constant $\varepsilon_{\mathrm{s}}$, density $\rho$, viscosity $\eta$ and refractive index $n_{D}$, of binary systems of DMSO with NM at $293.15 \mathrm{~K}$ over the entire composition range. Excess static dielectric constant, Bruggeman factor, excess density, excess viscosity $\eta^{\mathrm{E}}$, excess refractive index; have been calculated from the measured parameters. The calculated excess or deviation functions have been fitted to the Redlich-Kister polynomial equation.

\section{EXPERIMENTAL}

\subsection{Methods}

Dielectric Measurements: TDR Setup and Data acquisition: The complex permittivity spectra were studied using the time domain reflectometry (TDR) method as described in [1-4]. The Hewlett Packard HP 54750 sampling oscilloscope with HP54754A TDR plug in module has been used. Density Measurement: The Density measurements were carried out by Digital Density meter (Anton Paar -35) for pure liquids and binary mixture. Viscosity Measurements: Viscosity of the sample in the present study was measured by using Brookfield Viscometer, Model: LV DV-II+ Pro, Cone-plate Model with CPE-40 spindle. Refractive Index Measurements: The Refractive Index measurements are studied using Abbeys Refractometer

\section{RESULTS AND DISCUSSION}

Table 1. Density, Viscosity, Refractive index, Dielectric constant of NM+DMSO at 293.15K

\begin{tabular}{|c|c|c|c|c|}
\hline $\begin{array}{c}\text { Volume } \\
\text { fraction of } \\
\text { NM }\end{array}$ & $\begin{array}{c}\text { Density } \\
\left(\mathbf{g m} / \mathbf{c m}^{\mathbf{3}}\right)\end{array}$ & $\begin{array}{c}\text { Viscosity } \\
\mathbf{( c P )}\end{array}$ & RI & $\begin{array}{c}\text { Dielectric } \\
\text { constant }\end{array}$ \\
\hline 0 & 1.100 & 2.00 & 1.479 & 46.90 \\
\hline 0.1 & 1.102 & 1.78 & 1.478 & 45.78 \\
\hline 0.2 & 1.104 & 1.62 & 1.474 & 44.67 \\
\hline 0.3 & 1.109 & 1.48 & 1.469 & 43.56 \\
\hline 0.4 & 1.112 & 1.34 & 1.460 & 42.45 \\
\hline 0.5 & 1.110 & 1.15 & 1.452 & 41.33 \\
\hline 0.6 & 1.120 & 1.04 & 1.437 & 40.25 \\
\hline 0.7 & 1.124 & 0.93 & 1.418 & 39.15 \\
\hline 0.8 & 1.130 & 0.79 & 1.403 & 38.05 \\
\hline 0.9 & 1.134 & 0.66 & 1.391 & 36.95 \\
\hline 1 & 1.138 & 0.61 & 1.381 & 35.87 \\
\hline
\end{tabular}




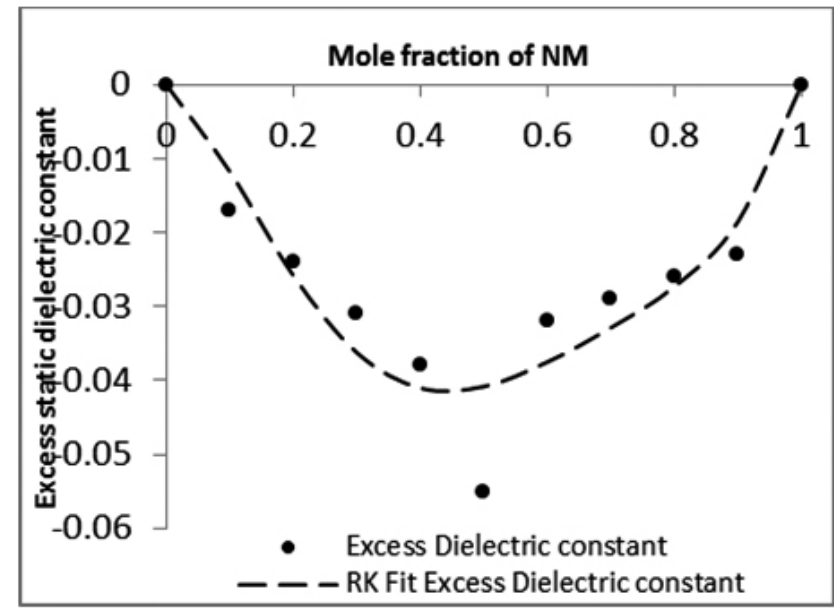

Fig 1 Excess static dielectric constant of DMSO+NM at 293.15K.

Fig 1 shows Negative deviation in excess static dielectric constant. The negative value of excess static dielectric constant indicates (Fig. 1) that the solute and solvent interact in such a manner so as to increase the effective dipole moment and leads to the formation of multimers. The negative excess static dielectric constant also indicates that one of the mixture constitute acts as a structure breaker for another molecule during the cooperation with dipoles of similar orientation. Hence there is decrease in the total number of parallel aligned effective dipoles that contribute to the mixture dielectric polarization.

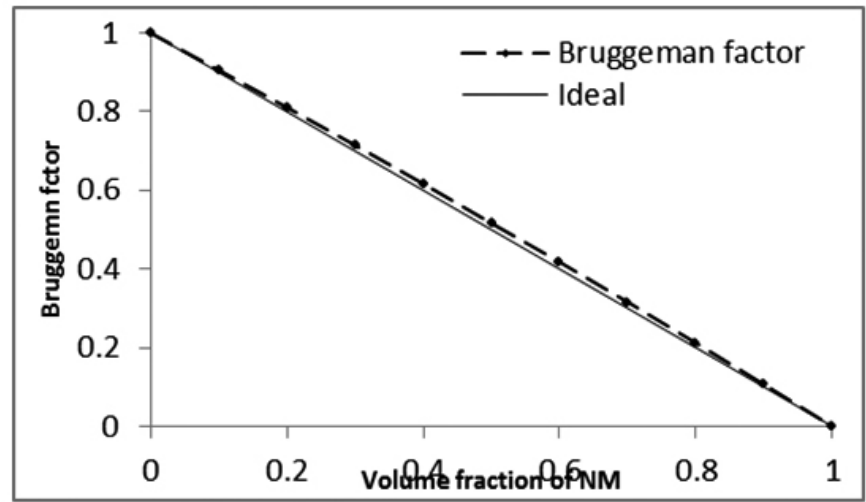

Fig 2 Bruggeman factor of DMSO+NM at 293.15 K.

Fig 2 shows deviation in ideal line of bruggeman plot. Deviation from ideal line indicates presence of intermolecular interactions within the system.

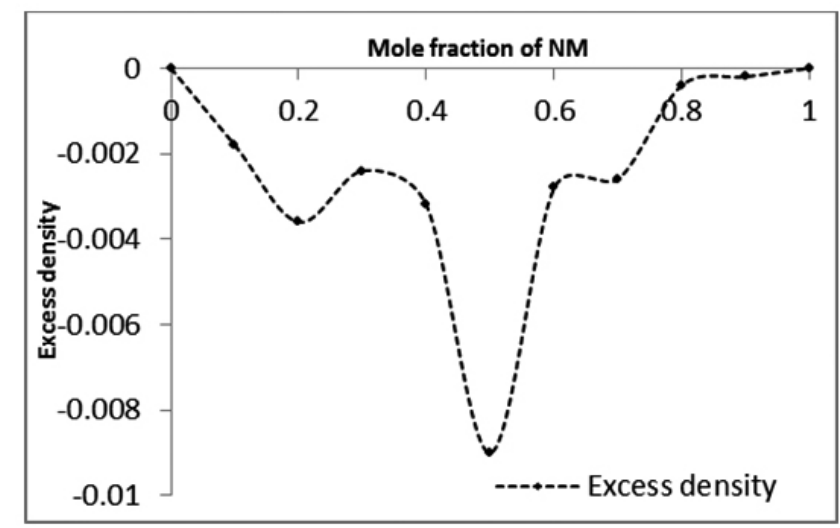

Fig 3 gives excess density of NM+DMSO.
Negative values of excess density show weak intermolecular interactions arised from dipole-dipole interaction. Negative values also indicate expansion of volume take place due to weak intermolecular interactions. This may happen as molecules of NM does not cooperate with molecules of DMSO and force of repulsion becomes dominant and hence expansion of liquid mixture takes place.

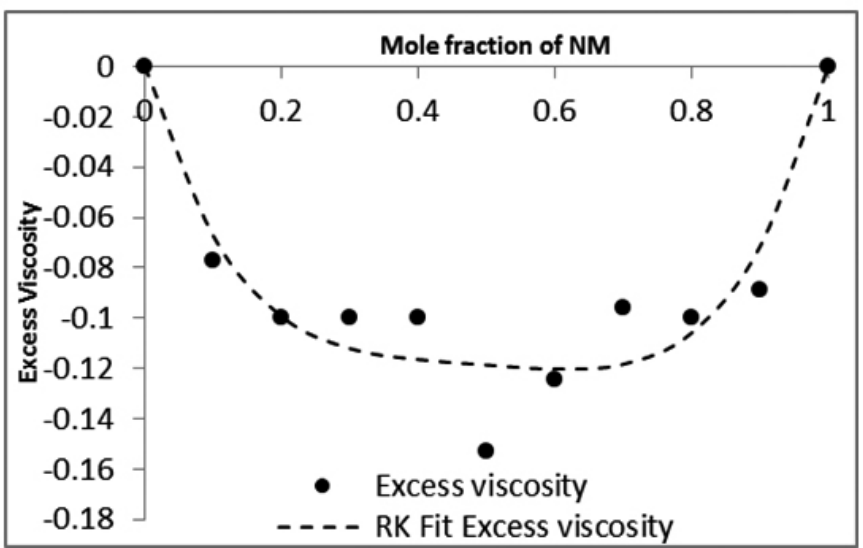

Fig 4 Excess Viscosity of NM+ DMSO at 293.15K.

Fig 4 gives Excess Viscosity of NM+DMSO. Negative values of $\eta^{\mathrm{E}}$ for the mixture can be explained on the basis of complex formation between unlike molecules. Negative values of excess viscosity for mixture may be attributed to presence of specific intermolecular interaction.

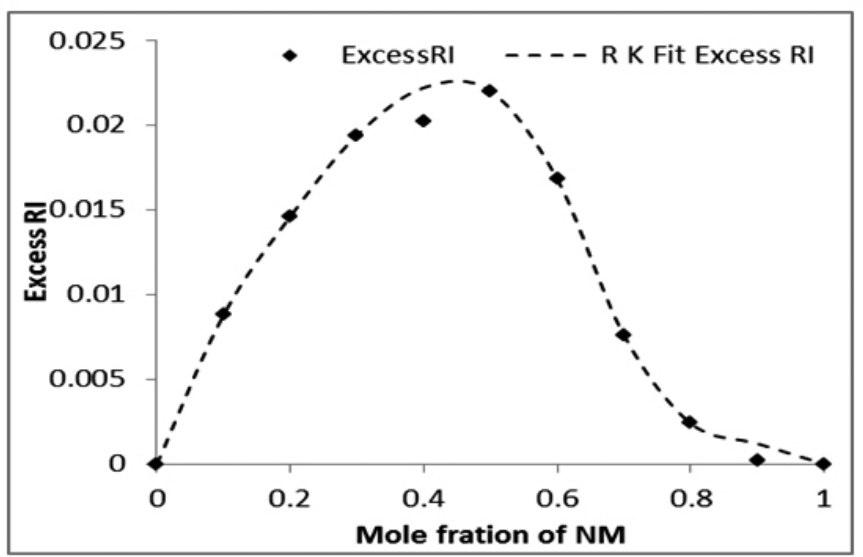

Fig 5 Excess refractive index of DMSO+NM at 293.15K.

Fig 5 shows Positive value of excess molar refraction indicates presence of specific intermolecular interaction arised from dipole-dipole or Vander walls interaction.

\section{CONCLUSIONS}

In this study, the measurement of density, viscosity, refractive index of $\mathrm{NM}$ in DMSO solution was studied in different concentrations at $293.15 \mathrm{~K}$. The experimental data and excess parameters contain valuable information regarding the solute-solvent interactions. It can be concluded that at 293.15 $\mathrm{K}$ concentration of the NM affects and gives rise to specific intermolecular interaction arised due to presence of weak dipole-dipole interactions between solute and solvent

\section{REFERENCES}

[1]. Aruna P. Maharolkar, Y S Sudke, S P Kamble A L Tidar, A G Murugkar, S S Patil, P W Khirade S C Mehrotra, Densities, Viscosities and Refractive Indices of n- Butanol + Allyl Chloride Mixture at 298K ,International Journal of Chemistry, Vol 2 No.2 250-260 , (2010). 
[2]. Aruna P. Maharolkar, A. G. Murugkar, S. S. Patil P. W. Khirade , Characterization of Dominant Hydrogen Bonded Complex Structures, Asian Journal of Chemistry, vol 25 No. 2 937-940,( 2013)

[3] Aruna P. Maharolkar, A. G. Murugkar And P. W. Khirade, Microwave Dielectric Characterization of Polar Protic liquids using Time Domain Reflectometry International journal of pharma and biosciences, 5 (2), 377$382,(2014)$.
[4] Aruna P Maharolkar, A G Murugkar, S S Patil, P W Khirade Characterization Of Interaction In Binary Mixtures by Dielectric Analysis International journal of pharma and biosciences, vol. 3,(4): 484-444, (2012)

[5] Aruna P Maharolkar, et al. Dielectric relaxation study of polar protic and aprotic polar solvent Asian Journal of Chemistry; 24, (12): 5680-5682, (2012). 